\title{
Conus Medullaris Syndrome following Spinal Cord Stimulator Trial: Pathomechanics and Troubleshooting Options
}

\author{
Gaurav Chauhan, MD, Daanish M. Khaja, MD, Himabindu Gandam Venkata, MD, \\ Joseph M Salama Hanna, MD, and Aman Y Upadhyay, MD
}

The conus medullaris is the lower bulbous section of the spinal cord where the cord begins to taper down. The tapering typically occurs around the first, and occasionally the second, lumbar $(\mathrm{L})$ vertebrae (1). This area typically incorporates the sacral (S) segments of the spinal cord. There is a multitude of etiologies of conus medullaris syndrome, including vertebral fractures, spinal trauma, neoplasms, infection, and herniated discs. Injuries or dysfunction of this area often results in a wide constellation of symptoms termed as conus medullaris syndrome (2). This area is a common location for the introduction of leads during dorsal column spinal cord stimulator (SCS) trial. SCS, a device used for neuromodulation of dorsal column pathways, is intended to reduce the afferent nociceptive activity within pain pathways. We present a case report of iatrogenic conus medullaris syndrome as a complication of lead placement during a neuromodulation trial.

\section{CASE REPORT}

A written consent was obtained from the patient for this case report. A 73-year-old male patient with a history of obesity (body mass index $34.64 \mathrm{~kg} / \mathrm{m}^{2}$ ), type II diabetes, essential hypertension, and lumbar spondylosis with radiculopathy, presented for treatment of low back pain. He reported that the pain was constant, with $8 / 10$ intensity, radiating to thighs bilaterally, aggravated by walking, standing, and lifting

From : Henry Ford Health System, Detroit, MI

Author for correspondence: Gaurav Chauhan, MD

Address: Department of Anesthesiology, Henry Ford Health System, Apt 1607, 1350 W. Bethune St., Detroit, MI 48202

E-mail: Gchauha1@hfhs.org heavy objects, and alleviated by sitting or lying flat. The symptoms were refractory to physical therapy and medical management. The patient underwent 2 lumbar surgeries, including a minimally invasive lumbar laminectomy followed by an L2-S1 fusion, which failed to alleviate his pain or improve his functionality. He subsequently developed intensified low back pain and was diagnosed with post laminectomy syndrome. The patient also reported poor response to his medication regimen of oxycodone $30 \mathrm{mg}$, every 6 hours as needed, and methocarbamol $750 \mathrm{mg}$ daily. The patient was using a walker to ambulate and occasionally using a wheelchair when the pain was severe.

The first SCS trial failed as there was no change in the characteristics of his pain and the decision was made to offer a second SCS trial utilizing high frequency stimulation. During the planned procedure, under fluoroscopic guidance, the interlaminar space between L1-L2 was identified. Using an anteroposterior and lateral fluoroscopic approach, a 4.5 in, 14-gauge, curved Tuohy epidural needle was guided into the interlaminar space. Subsequently, using the loss-of-resistance technique, the epidural space was identified and the SCS lead was placed on the right side in the dorsal portion of the epidural space. The right-sided lead was then advanced without difficulty up to the level of T6. The patient reported a good response from the right SCS lead stimulation.

Employing the same methodological approach, an attempt was made to place a SCS lead on the left in the L1-L2 epidural space. After guiding the lead up to the 11th thoracic vertebrae, we were unable to secure the lead in the dorsal position and subsequent stimulation of the lead resulted in paresthesia of the anterior abdomen. After repeated unsuccessful attempts, the lead was removed and the procedure was abandoned. The patient was moved to the recovery 
area where he complained of severe right scrotal and perianal pain. He was given a single bolus of $50 \mathrm{mcg}$ of fentanyl, which provided no relief. He had intact sensation of his bowels and could control the external anal sphincter, but an ultrasound of the bladder a few hours later revealed urinary retention with a bladder volume of $850 \mathrm{~mL}$. The patient denied any weakness, headaches, or difficulty walking. He was immediately transferred to the emergency department for further evaluation. Physical examination revealed normal reflexes and magnetic resonance imaging (MRI) was negative for any type of acute process such as a hematoma. In lieu of excruciating pain refractory to medical management, the decision was made to remove the right epidural lead. A neurosurgical consult ruled out cauda equina syndrome and the need for any emergent surgical intervention. Post removal of the right epidural lead and administration of opioid analgesics (fentanyl $100 \mathrm{mcg}$, intravenously) the patient reported tolerable pain scores. The patient was subsequently discharged home and a close follow-up appointment was scheduled. The patient was prescribed nortriptyline, which helped in mitigating the pain. The scrotal and perianal pain resolved over the next 6 weeks and there was no further incidence of urinary retention.

\section{DISCUSSION}

SCS was approved as a treatment modality for chronic pain diagnosis such as radiculopathies refractory to conservative or surgical treatment, peripheral neuropathies, complex regional pain syndrome, etc (3). Current literature reports that SCS implantation is associated with adverse events in 1 in 3 subjects (4). Furthermore, a recent closed claims review reported that one of the most common device related claims were due to SCS implantation ( $n=43,29 \%)$ with trauma to cord or cauda equina reported in $9 \%$ $(n=10)$ subjects $(5)$.

Conus medullaris syndrome is a presentation of neurologic dysfunction that is difficult to differentiate from other spinal cord pathology such as cauda equina syndrome. The immediate challenge faced by the authors, in this case, was to rule out cauda equina syndrome. The pathological basis of conus medullaris syndrome is an insult to the distal bulbous part of the spinal cord at the level of L1-L2, which is comprised of sacral (S1-S5) spinal nerves, affecting both upper and lower motor neurons. In contrast, cauda equina syn- drome is a lesion of lumbosacral nerve roots leading to lower motor neurons symptomatology only. Clinically conus medullaris syndrome may encompass signs such as perianal sensory loss (S3-S5), absent bulbocavernosus reflexes (S2-S4), and normal or increased rectal tone (S2-S5) along with symmetric and hyperreflexic distal paresis of lower extremities, specifically in muscles supplied by S1-S5 (6). The symptoms include back pain, sudden unilateral or bilateral radicular pain at the L1-L2 dermatomal level, along with an early onset of bladder, bowel, and sexual dysfunction in contrast to cauda equina syndrome, where the urinary and fecal incontinence are usually less severe in magnitude and frequently a late finding $(7,8)$.

In cauda equina syndrome the radicular pain is gradual in onset, usually unilateral and comparatively more severe in L2-S5 dermatomal levels. There is asymmetric anesthesia of S3, S4, and S5 dermatomal levels, involving anal, perineal, and genital regions, leading to the sensory loss in the saddle region, which can extend to the dorsal aspect of the thigh, anterolateral aspect of the leg and the outer aspect of the foot (9). With extensive lesions, patients can develop flaccid, hypotonic, areflexia, and paralysis that affects the glutei, thigh muscles, and anterolateral muscles of the leg and foot. Muscle strength testing of the hip flexors (iliopsoas, L2), knee extensors (quadriceps, L3), ankle dorsiflexor (tibialis anterior, L4), big toe extensors (extensor hallucis long, L5), and ankle plantar flexors (gastrocnemius/soleus, S1) can further give insight into the level of injury $(10,11)$.

The immediate management of a patient with conus medullaris syndrome is directed at early identification of the primary cause of the symptoms. A prompt evaluation of signs and symptoms experienced by the patient and a focused neurological examination is vital for diagnosis and to determine further intervention (12). The prognosis improves significantly if the definitive cause is identified and relevant treatment is instituted promptly $(13,14)$. The subject in this case report had typical symptoms of conus medullaris syndrome, which was high on the list of the authors' differentials. After a thorough review of the events, the authors unanimously agreed that compression of the thecal sac by the SCS lead in the epidural space causing neuroparaxia may be the primary cause of the patient's distressing signs and symptoms (Fig. 1). 


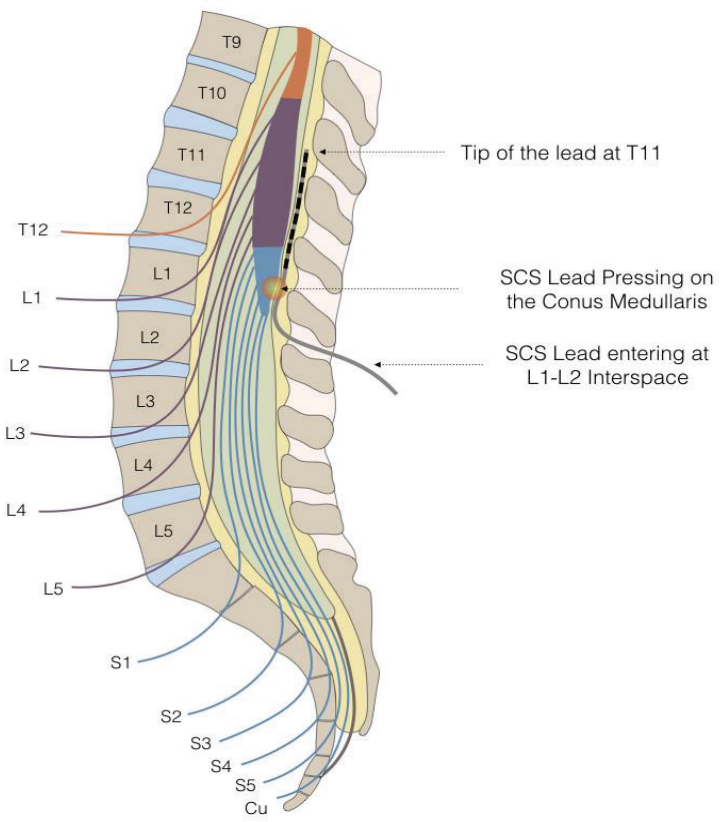

Fig. 1. An illustration of possible injury caused by SCS leads.

The lead was subsequently removed leading to resolution of the signs and symptoms. The diagnosis was further aided by early consultation with neurosurgery. The MRI is urgently indicated in patients who develop new onset urinary symptoms associated with back pain and is the preferred imaging modality of choice to document the degree of neurological involvement (15). An MRI scan, due to inherently superior contrast resolution, is more sensitive than other imaging modalities in localizing the site of the lesion. As compared to the computerized tomographic scan (CT), that classifies the neurological injury according to the vertebral level, the MRI scan delineates the soft tissue anatomy, shedding more light on the etiology and accurately identifies the level of neuraxial insult, which has been shown to correlate with patient outcomes (16). The MRI scan didn't report any compression or edema of conus medullaris, but there is a possibility that the static images failed to capture the dynamic pathology of the spine (Figs. 2 and 3). Ultrasonography examination of the urinary bladder may be used to estimate or measure post void residual bladder volume. Furthermore, urodynamic studies may be used to evaluate the degree of sphincter dysfunction,

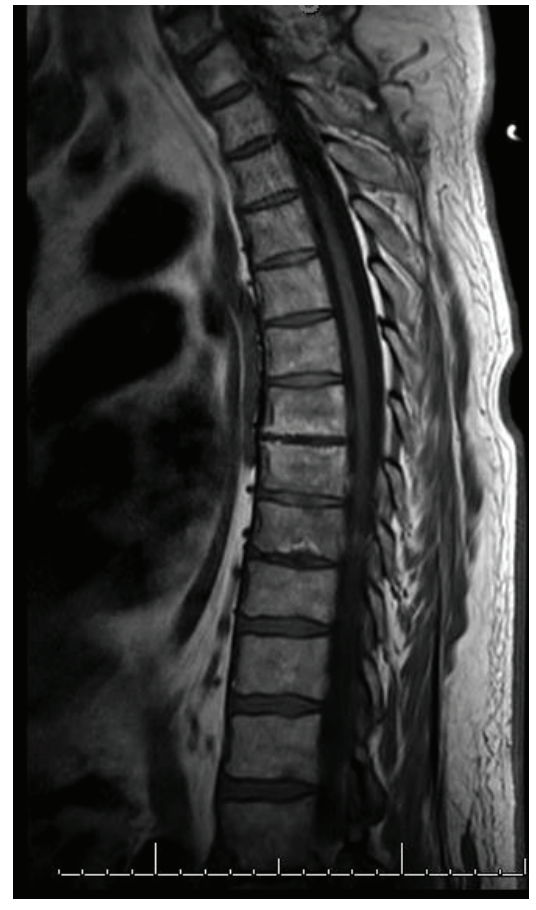

Fig. 2. T1 weighted MRI of the thoracic spine. Sagittal section reporting chronic multilevel degenerative changes of thoracic spine.

as well as to monitor recovery of bladder function following an intervention (17). A series of close followup appointments should be scheduled to peruse the patient and provide a panacea for adverse sequelae or relapse of symptoms until the patient returns back to baseline. There is insufficient evidence in the current literature regarding the natural history of conus medullaris syndrome, however, patient's age, adequacy of vascular supply and magnitude and the neuraxial level of injury are some of the factors that come into play while predicting the outcome. The current literature is inexplicit and outcomes are less predictable as far as the operative intervention for conus medullaris syndrome is concerned, however, some studies have reported improved functional outcomes if surgical decompression is done within 48 hours. Furthermore, knowledge is sparse regarding the natural course of conus medullaris syndrome and poor outcomes with permanent neurological damage are reported despite operative or non-operative 


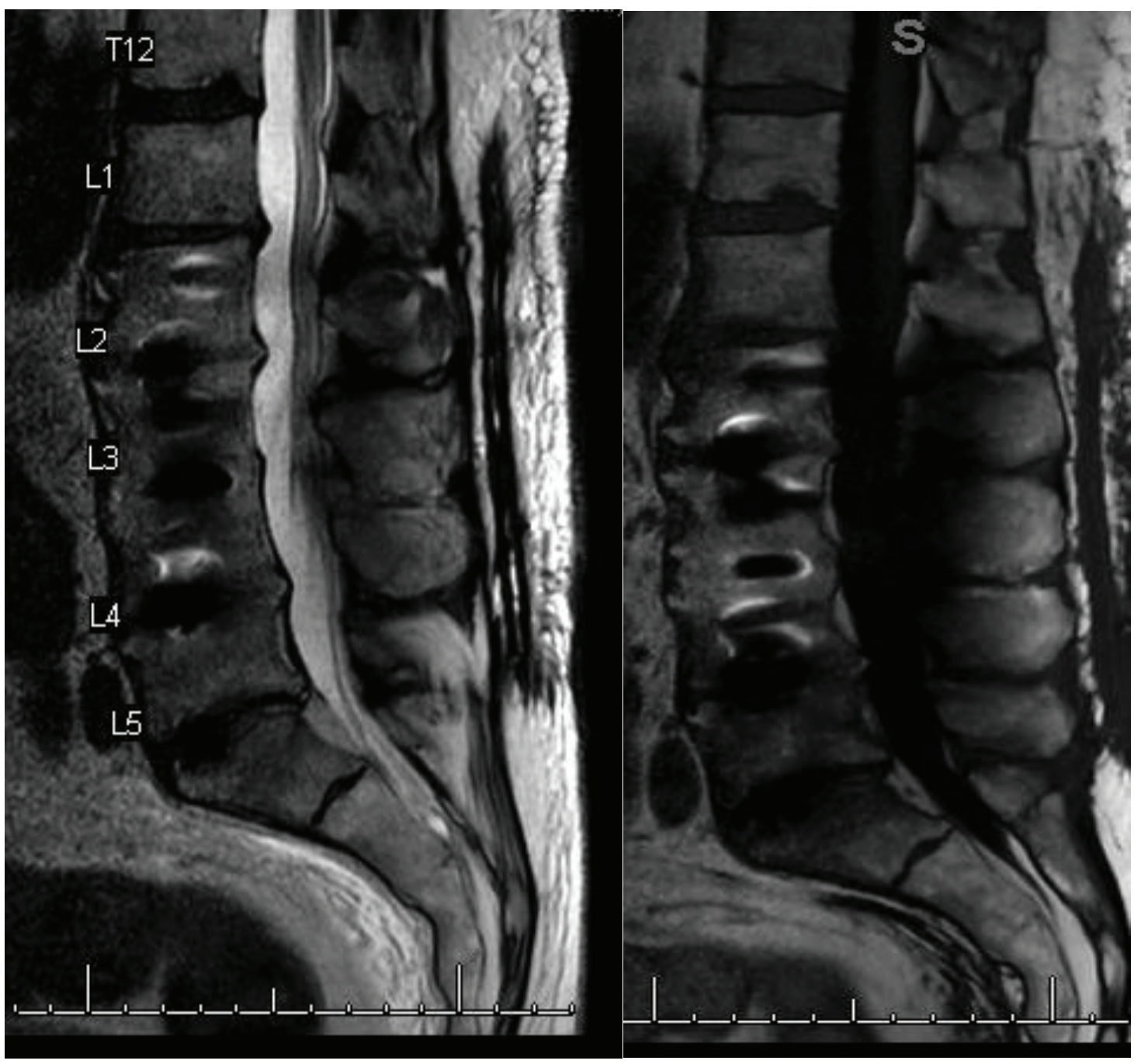

Fig. 3. T1 and T2 weighted MRI of the lumbar spine. Sagittal section reporting postsurgical and multilevel chronic degenerative changes of the lumbar spine. The conus medullaris ends normally at the L1 level and there is no evidence of focal enlargement or intramedullary signal abnormality.

management $(8,14)$. The subject in this case report was neurologically stable, however in patients with progressive neurological decline or objective MRI evidence of cord compression, surgical decompression is the logical choice.

This is the first reported case of conus medullaris syndrome secondary to SCS lead placement. This case report emphasizes the fact that a high degree of vigilance should be maintained when introducing foreign objects into the spinal column during interventional pain procedures. In such a scenario, the physician should be able to identify the cause, differentiate between conus medullaris and cauda equina syndrome, and should not hesitate to involve the relevant subspecialties to aid the diagnosis. It is of paramount importance to adequately counsel, reassure, and explain to the patient about the course of this phenomenon to allay anxiety and any increased emotional suffering. 
Conus Medullaris Syndrome following SCS

\section{REFERENCES}

1. Mauffrey C, Randhawa K, Lewis C, Brewster M, Dabke H. Cauda equina syndrome: An anatomically driven review. $\mathrm{Br}$ J Hosp Med (Lond) 2008; 69:344-347.

2. Rogers WK, Todd M. Acute spinal cord injury. Best Pract Res Clin Anaesthesiol 2016; 30:27-39.

3. American Society of Anesthesiologists Task Force on Chronic Pain Management, Practice guidelines for chronic pain management: An updated report by the American Society of Anesthesiologists Task Force on Chronic Pain Management and the American Society of Regional Anesthesia and Pain Medicine. Anesthesiology 2010; 112:810-33.

4. Turner, JA, Loeser, JD, Deyo, RA, Sanders, SB. Spinal cord stimulation for patients with failed back surgery syndrome or complex regional pain syndrome: A systematic review of effectiveness and complications. Pain 2004; 108:137-147.

5. Fitzgibbon DR, Stephens LS, Posner KL, Michna E, Rathmell JP, Pollak KA, Domino KB. Injury and liability associated with implantable devices for chronic pain. Anesthesiology 2016; 124:1384-1393.

6. Kennard C. Neurology in clinical practice. Principles of diagnosis and management. Journal of the Royal Society of Medicine 1991; 84:574.

7. Bardin LD, King P, Maher CG. Diagnostic triage for low back pain: A practical approach for primary care. Med J Aust 2017; 206:268-273.

8. Harrop JS, Hunt GE Jr, Vaccaro AR. Conus medullaris and cauda equina syndrome as a result of traumatic injuries: Management principles. Neurosurg Focus 2004; 16:e4.
9. McKinley W, Santos K, Meade M, Brooke K. Incidence and outcomes of spinal cord injury clinical syndromes. J Spinal Cord Med 2007; 30:215-224.

10. Rydevik B. Neurophysiology of cauda equina compression. Acta Orthop Scand Suppl 1993; 251:52-55.

11. Olivero WC, Wang H, Hanigan WC, Henderson JP, Tracy PT, Elwood PW, Lister, JR, Lyle L. Cauda equina syndrome (CES) from lumbar disc herniations. J Spinal Disord Tech 2009; 22:202206.

12. Todd NV. An algorithm for suspected cauda equina syndrome. Ann R Coll Surg Engl 2009; 91:358-359.

13. Ahn UM, Ahn NU, Buchowski JM, Garrett ES, Sieber AN, Kostuik JP. Cauda equina syndrome secondary to lumbar disc herniation: A meta-analysis of surgical outcomes. Spine (Phila Pa 1976) 2000; 25:1515-1522.

14. Kingwell SP, Curt A, Dvorak MF. Factors affecting neurological outcome in traumatic conus medullaris and cauda equina injuries. Neurosurg Focus 2008; 25:E7.

15. Coscia M, Leipzig T, Cooper D. Acute cauda equina syndrome. Diagnostic advantage of MRI. Spine (Phila Pa 1976) 1994; 19:475-478.

16. Parizel PM, van der Zijden T, Gaudino S, Spaepen M, VoormoIen MHJ, Venstermans C, DeBelder F, Van Den Hauwe L, Van Goethem J. Trauma of the spine and spinal cord: Imaging strategies. European Spine Journal 2010; 19:8-17.

17. Gleave JR, MacFarlane R. Prognosis for recovery of bladder function following lumbar central disc prolapse. Br ] Neurosurg 1990; 4:205-209. 
